\title{
Microscopic model of Purcell enhancement in hyperbolic metamaterials
}

\author{
Alexander N. Poddubny, ${ }^{1,2}$ Pavel A. Belov, ${ }^{1,3}$ Pavel Ginzburg, ${ }^{4}$ Anatoly V. Zayats, ${ }^{4}$ and Yuri S. Kivshar ${ }^{1,5}$ \\ ${ }^{1}$ National Research University for Information Technology, Mechanics and Optics (ITMO), St. Petersburg 197101, Russia \\ ${ }^{2}$ Ioffe Physical-Technical Institute of the Russian Academy of Science, St. Petersburg 194021, Russia \\ ${ }^{3}$ School of Electronic Engineering and Computer Science, Queen Mary University of London, London E1 4NS, United Kingdom \\ ${ }^{4}$ Department of Physics, King's College London, London WC2R 2LS, United Kingdom \\ ${ }^{5}$ Nonlinear Physics Center and Center for Ultrahigh-Bandwidth Devices for Optical Systems (CUDOS), Research School of Physics \\ and Engineering, Australian National University, Canberra, Australian Capital Territory 0200, Australia
}

(Received 16 May 2012; revised manuscript received 11 July 2012; published 30 July 2012)

\begin{abstract}
We study theoretically the dramatic enhancement of spontaneous emission in metamaterials with the hyperbolic dispersion modeled as a cubic lattice of anisotropic resonant dipoles. We analyze the dependence of the Purcell factor on the source position in the lattice unit cell and demonstrate that the optimal emitter positions needed to achieve large Purcell factors and Lamb shifts are in the local field maxima. We show that the calculated Green function has a characteristic crosslike shape, spatially modulated due to the structural discreteness. Our basic microscopic theory provides fundamental insights into the rapidly developing field of hyperbolic metamaterials.
\end{abstract}

DOI: 10.1103/PhysRevB.86.035148

PACS number(s): 42.50.-p, 74.25.Gz, 78.70.-g

\section{INTRODUCTION}

The enhancement of the radiative decay rate of a source placed in a microwave resonant cavity was first discussed by Purcell in 1946 (Ref. 1) and is generally called the Purcell effect. ${ }^{2}$ Engineering and enhancement of the lightmatter interaction in a nanostructured environment is the focus of active research. ${ }^{3-9}$ In particular, the so-called hyperbolic metamaterials have attracted significant attention. ${ }^{10-12}$ Realizations of the regime of a hyperbolic medium with negative components of the dielectric tensor have been reported for magnetized plasma, ${ }^{13}$ graphite, ${ }^{14}$ metamaterials based on nanorod assemblies ${ }^{15-17}$ and layered metal-dielectric structures. ${ }^{4,18,19}$ In particular, hyperbolic dispersion can appear when nanostructured media composed of small elementary units are described within the effective-medium approximation as a uniaxial material with the main components of the effective dielectric or magnetic tensors of different sign. In this regime, light wave vectors at a given frequency fill a surface of hyperbolic shape, so that the area of the hyperbolic isofrequency surface, giving the photonic density of states, is infinite. As a result, the spontaneous emission rate becomes infinite in an ideal hyperbolic medium. ${ }^{4,20}$

The detailed study of the Purcell enhancement of the spontaneous emission rate in hyperbolic metamaterials has attracted special attention most recently in view of a series of experimental demonstrations. ${ }^{4,21-23}$ A number of theoretical studies have also been performed for various models. ${ }^{24}$ In particular, it has been shown that the Purcell factor should not actually diverge, since it is determined by a cutoff in the wave-vector space, stemming from spatial inhomogeneity of the medium, ${ }^{25-27}$ the finite distance from the source to the medium, ${ }^{28-30}$ nonlocality of the dielectric response, ${ }^{31}$ or the finite size of the emitter. ${ }^{32}$

Since the basic solid-state model of either natural or artificial materials is a periodic lattice of unit cells, here we adopt this model and consider a hyperbolic material as an infinite cubic crystal of interacting resonant point dipoles. Similar models have been developed for various systems including lattices of quantum dots, ${ }^{33,34}$ optical atomic lattices, ${ }^{35-38} \gamma$-ray resonant nuclear scattering, ${ }^{39}$ and the discrete-dipole approximation of light scattering theory. ${ }^{40,41}$ This general approach, despite certain limitations, has also been applied to lattices of split-ring resonators. ${ }^{42-46}$

In this paper, we study optical properties of an infinite cubic crystal of resonant interacting point dipoles polarizable only in one direction (see Fig. 1). This model allows us to reproduce the hyperbolic isofrequency surfaces of uniaxial anisotropic metamaterials and accounts for the discrete character of metamaterials. Within this microscopic model of a hyperbolic metamaterial, we investigate the influence of emitter position within the unit cell of the metamaterial on its radiation properties.

The paper is organized as follows. Section II outlines our theoretical model and approach. The calculated dispersion and lattice Green function are discussed in Sec. III. Section IV is devoted to the numerical and analytical results on the Purcell factor and Lamb shift in metamaterials with hyperbolic dispersion.

\section{DISCRETE-DIPOLE MODEL}

We consider an infinite periodic cubic lattice $\boldsymbol{r}_{j}$ of point dipoles, characterized by the period $a$, and embedded in vacuum. Our approach can be straightforwardly generalized to allow for a background dielectric constant $\varepsilon \neq 1$. The emitter inside the lattice is modeled by a radiating dipole $\boldsymbol{p}_{0}$ placed in the point $\boldsymbol{r}_{0}$. The structure's geometry is sketched in Fig. 1 . The self-consistent electric field satisfies the following equation:

$$
\nabla \times \nabla \times \boldsymbol{E}-q^{2} \boldsymbol{E}=4 \pi q^{2} \boldsymbol{P}
$$

where $q=\omega / c$ is the wave vector at the frequency $\omega$. The quantity $\boldsymbol{P}$ in Eq. (1) is the net polarization of the lattice dipoles and the emitter:

$$
\boldsymbol{P}=\boldsymbol{d}_{0} \delta\left(\boldsymbol{r}-\boldsymbol{r}_{0}\right)+\sum_{j} \boldsymbol{p}_{j} \delta\left(\boldsymbol{r}-\boldsymbol{r}_{j}\right)
$$




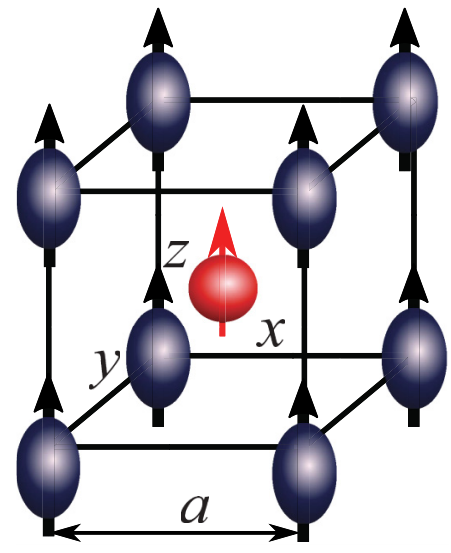

FIG. 1. (Color online) Schematic illustration of the unit cell of the cubic dipole lattice with embedded light source.

All the dipoles $\boldsymbol{p}_{j}$ are characterized by the identical polarizability tensor $\hat{\alpha}$ :

$$
\boldsymbol{p}_{j}=\hat{\alpha} \boldsymbol{E}_{\text {ext }}\left(\boldsymbol{r}_{j}\right) .
$$

Split rings, being the building blocks of metamaterials, should be generally described by both magnetic and electric dipole moments; see the comprehensive discussion in Ref. 47. However, in the simplest approximation one can retain only the magnetic polarizability, and the problem becomes equivalent to the one considered here. ${ }^{42}$ Analytical values of the polarizability for atoms and small dielectric or plasmonic spheres are given, e.g., in Ref. 48, and the resonant polarization of quantum dots is discussed in Ref. 7.

Our goal is to determine the total electric field and polarizations induced in the structure by the radiating dipole $\boldsymbol{d}_{0}$. This procedure includes field expansion over the Bloch eigenmodes with wave vectors $\boldsymbol{k}$, for which Eqs. (1) and (2) are independent. The results in a coordinate space are obtained by inverse Fourier transformation. In particular, the polarizations of the lattice dipoles read

$$
\boldsymbol{p}_{j}=\int_{(\mathrm{BZ})} \frac{V_{0} d^{3} k}{(2 \pi)^{3}} e^{i \boldsymbol{k} \cdot \boldsymbol{r}_{j}} \hat{\alpha}[\hat{\imath}-\hat{C}(\boldsymbol{k}) \hat{\alpha}]^{-1} \hat{G}_{0, \boldsymbol{k}}\left(-\boldsymbol{r}_{0}\right) \boldsymbol{d}_{0},
$$

where $V_{0}=a^{3}$ is the unit cell volume, the integration takes place over the Brillouin zone (BZ), $\left|k_{m}\right|<\pi / a, m=x, y, z$, and $\hat{1}$ is a $3 \times 3$ unit matrix. The radiating dipole position $\boldsymbol{r}_{0}$ enters Eq. (4) and thus determines the efficiency of the lattice excitation. The quantity $\hat{C}$ in Eq. (4) is the tensor interaction constant of the lattice, defined as ${ }^{43}$

$$
\hat{C}(\boldsymbol{k})=\lim _{\boldsymbol{r} \rightarrow \mathbf{0}}\left[\hat{G}_{0, \boldsymbol{k}}(\boldsymbol{r})-\hat{G}_{0}(\boldsymbol{r})\right]+\frac{2 i q^{3}}{3} \hat{1},
$$

where $\hat{G}_{0, \boldsymbol{k}}$ is the Green function of the photon with Bloch vector $\boldsymbol{k}$,

$$
\hat{G}_{0, \boldsymbol{k}}(\boldsymbol{r})=\sum_{j} \hat{G}_{0}\left(\boldsymbol{r}-\boldsymbol{r}_{j}\right) e^{i \boldsymbol{k} \cdot \boldsymbol{r}_{j}}
$$

and $\hat{G}_{0}$ is the free-photon Green function

$$
\hat{G}_{0}(\boldsymbol{r})=\left[q^{2}+\nabla \nabla\right] \hat{1} \frac{e^{i q r}}{r} .
$$

The infinite lattice sums (6) may be found either by the Ewald summation ${ }^{49}$ or by the Floquet-type summation. ${ }^{43,50}$ We have used the approach from Ref. 43, since it is preferential for fast evaluation of the integral in Eq. (4). The electric field in the structure is given by the sum of the waves emitted by all the dipoles,

$$
\boldsymbol{E}(\boldsymbol{r})=\hat{G}_{0}\left(\boldsymbol{r}-\boldsymbol{r}_{0}\right) \boldsymbol{p}_{0}+\sum_{j} \hat{G}_{0}\left(\boldsymbol{r}-\boldsymbol{r}_{j}\right) \boldsymbol{p}_{j} .
$$

Equation (8) by definition provides the Green function for the source embedded in the dipole lattice. The second term in Eq. (8) is given by Eq. (4) where $e^{i \boldsymbol{k} \cdot \boldsymbol{r}_{j}}$ is replaced by $\hat{G}_{0, \boldsymbol{k}}(\boldsymbol{r})$.

From now we restrict ourselves to the case of uniaxial dipoles, when the only nonzero component of the tensor $\hat{\alpha}$ is $\alpha_{z z}$. We assume that the radiating dipole $\boldsymbol{d}_{0}$ is also directed along the $z$ axis. The TM-polarized Bloch eigenmodes of the system with given wave vector $\boldsymbol{k}$ are found ${ }^{43,45}$ from the poles of Eq. (4)

$$
\frac{1}{\alpha_{z z}}-C(\boldsymbol{k})=0,
$$

where $C(\boldsymbol{k}) \equiv C_{z z}(\boldsymbol{k})$. Note that Eq. (9) is real for vanishing losses, because the imaginary part of the interaction constant (5) cancels out with the radiative decay term in the polarizability:

$$
\frac{1}{\alpha_{z z}}=\frac{1}{\alpha_{0, z z}}-\frac{2 i q^{3}}{3} .
$$

Here, $\alpha_{0, z z}$ is the so-called bare-dipole polarizability calculated neglecting radiative decay. ${ }^{48}$ The effective-medium approximation for the solutions of Eq. (9) are the extraordinary TM-polarized modes with the dispersion given by ${ }^{51}$

$$
q^{2}=\frac{k_{x}^{2}+k_{y}^{2}}{\varepsilon_{z z}}+k_{z}^{2} .
$$

Here, $\varepsilon_{z z}$ is the Maxwell-Garnett effective dielectric constant of the hyperbolic medium

$$
\varepsilon_{z z}=1+\frac{1}{V /\left(4 \pi \alpha_{0, z z}\right)-1 / 3},
$$

in the same approximation $\varepsilon_{x x}=\varepsilon_{y y}=1$.

\section{DISPERSION AND GREEN FUNCTION}

In this section we first discuss the dispersion of the Bloch waves in the dipole lattice (Sec. III A) and then investigate in detail the emission pattern of the dipole embedded in the lattice (Sec. III B).

\section{A. Isofrequency curves}

Isofrequency curves in the $\left(k_{z}, k_{x}\right)$ plane, found from Eq. (9) for different polarizabilities $\alpha_{0, z z}$, are shown in Fig. 2. Depending on the polarizability, the dispersion curves are either elliptic or hyperbolic, in agreement with Eq. (12). The curves are generally well described by the effective-medium approximation Eq. (11). However, an intermediate "mixed" regime is possible for $\alpha_{0, z z} \approx-1.3 a^{3} /(4 \pi)$ (blue dashed curve), when two Bloch modes with hyperbolic and elliptic dispersion coexist in the structure. Such isofrequency curves cannot be described by the Maxwell-Garnett theory Eqs. (11) 


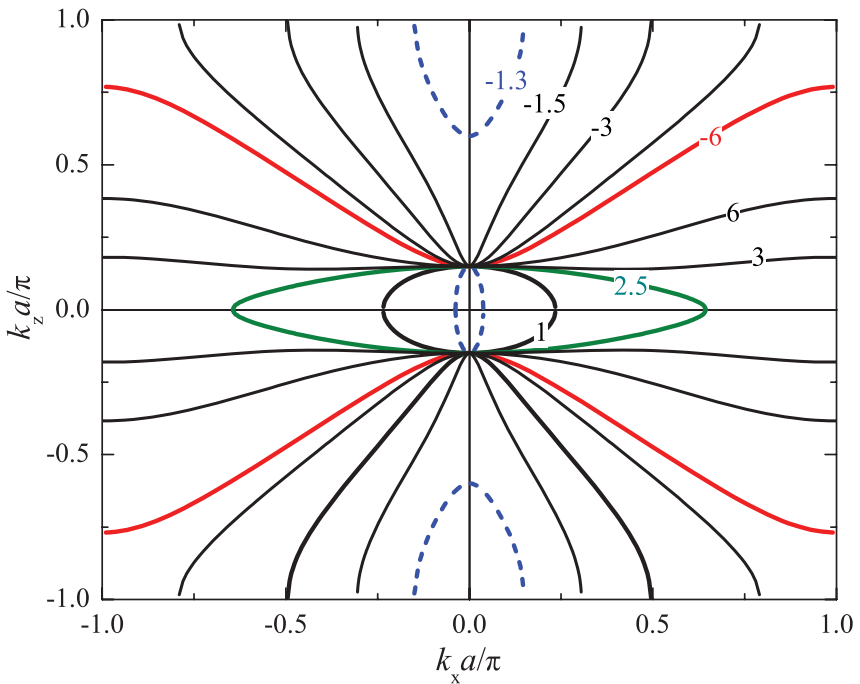

FIG. 2. (Color online) Isofrequency curves in the $x z$ plane, calculated for different dipole polarizabilities $\alpha_{0, z z}$. The normalized polarizability $4 \pi \alpha_{0, z z} / a^{3}$ is indicated near each curve. The calculation was performed at $q a=0.15 \pi$.

and (12), which predicts only one TM mode for a given frequency. They were analyzed in Ref. 43 in more detail and can be obtained in the effective-medium model when nonlocal effects are taken into account. ${ }^{52}$

\section{B. Green function}

Here we will focus on the spatial distribution [Eq. (4)] of the dipole moments $\left|p\left(\boldsymbol{r}_{j}\right)\right|$ in the discrete lattice under the pointdipole excitation. The results of calculations for the dipole polarizabilities $\alpha_{0, z z}=a^{3} /(4 \pi)$ and $\alpha_{0, z z}=-6 a^{3} /(4 \pi)$, corresponding to the elliptic and hyperbolic regimes, are shown in Figs. 3 and 4, respectively. The calculation demonstrates that the distribution is qualitatively different in the hyperbolic regime: the pattern is strongly anisotropic and has a characteristic crosslike shape; see Fig. 4. Moreover, in the hyperbolic

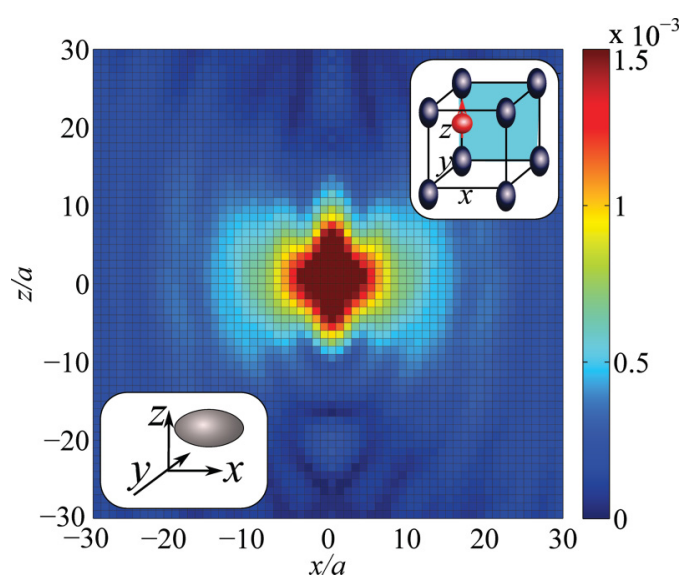

FIG. 3. (Color online) Spatial distribution of the dipole moments $\left|p_{z}(\boldsymbol{r})\right| / p_{0}$ in the elliptic regime with $\alpha_{0, z z}=a^{3} /(4 \pi)$. The insets schematically illustrate the geometry and the isofrequency surfaces in wave-vector space. The calculation was performed at $q a=0.15 \pi$ and $\boldsymbol{r}_{0}=0.5 a \hat{z}$.
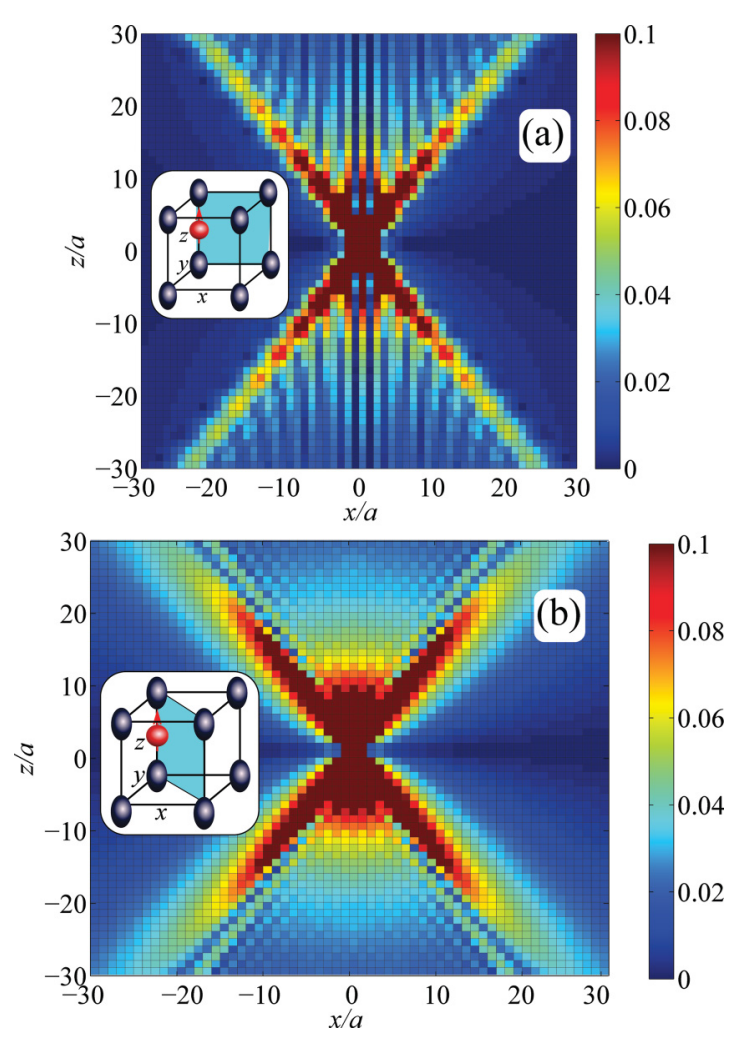

FIG. 4. (Color online) Spatial distribution of the dipole moments $\left|p_{z}(\boldsymbol{r})\right| / p_{0}$ in the hyperbolic regime, excited by the point emitter. (a) and (b) show the distributions in the planes $y=0$ and $x=y$, respectively. The insets schematically illustrate the geometry and the isofrequency surfaces in wave-vector space. The calculation was performed at $\alpha_{0, z z}=-6 a^{3} /(4 \pi)$ and the same other parameters as in Fig. 3 .

case, the pattern depends on the azimuthal direction: it has distinct vertical ripples in the plane $y=0$ [Fig. 3(a)], which are absent in the plane $y=x$ [Fig. 3(b)].

To understand these results it is instructive to compare them with the Green function in the effective-medium approximation (Fig. 5). This approximation allows one to obtain the solution in a closed form. ${ }^{10,53}$ In the case of a vertical orientation of the radiating dipole, $p_{0} \| z$, the Green function reads

$$
\begin{aligned}
\boldsymbol{E}_{\mathrm{eff}}(\boldsymbol{r}) & =\left(q^{2}+\nabla \nabla\right) p_{0} \hat{z} \frac{e^{i q R}}{R}, \\
R & =\sqrt{\varepsilon_{z z}\left(x-x_{0}\right)^{2}+\varepsilon_{z z}\left(y-y_{0}\right)^{2}+\left(z-z_{0}\right)^{2}} .
\end{aligned}
$$

This is a generalization of Eq. (7) in the case of a uniaxial medium. The relation between the electric field and the polarization in the effective-medium model is local,

$$
4 \pi \boldsymbol{P}_{\mathrm{eff}}=\left(\varepsilon_{\mathrm{eff}}-1\right) \boldsymbol{E}_{\mathrm{eff}} .
$$

It should be stressed that the effective-medium approximation is not applicable on spatial scales smaller than the lattice constant $a$. Consequently, the problem of a point radiating dipole in a discrete structure cannot be reduced to the effectivemedium one. The effects of the radiating dipole position within the unit cell are also beyond the effective-medium 

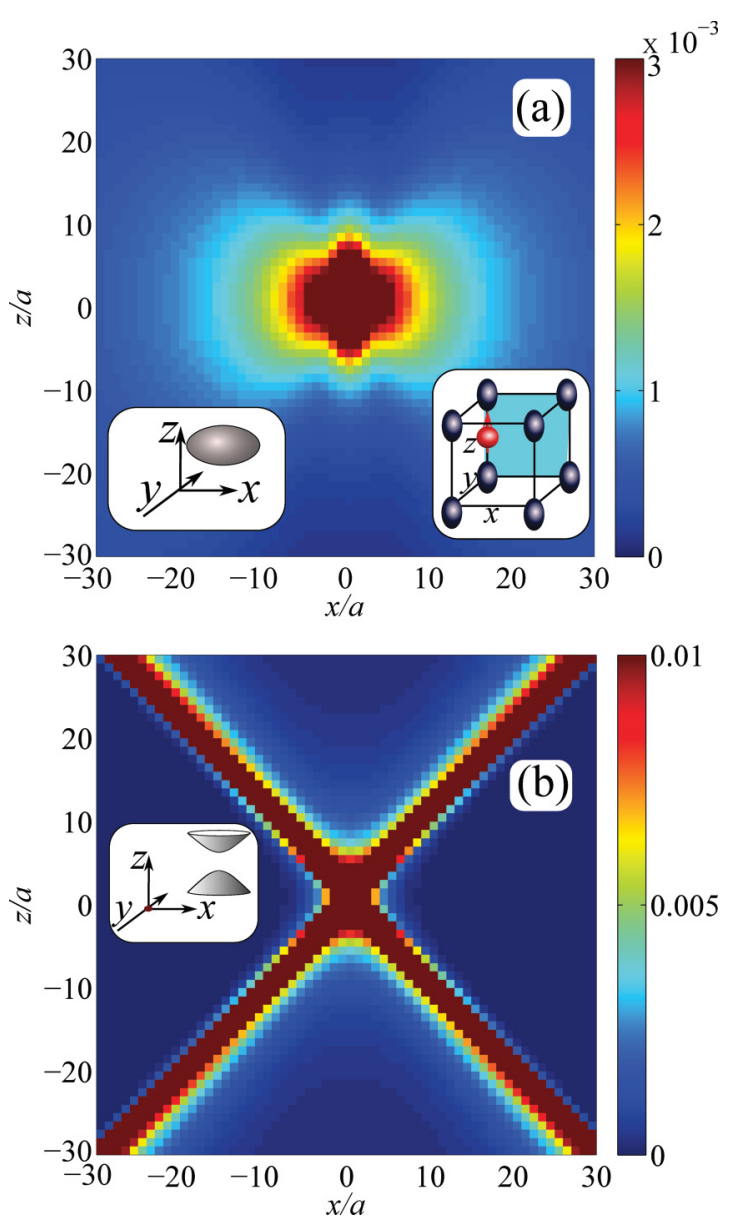

FIG. 5. (Color online) Spatial distribution of the polarization $P_{\text {eff }, z}(\boldsymbol{r}) /\left(p_{0} a^{3}\right)$ induced by the point source in (a) an effective elliptic medium with $\varepsilon_{z z}=2.5$ and (b) an effective hyperbolic medium with $\varepsilon_{z z}=-1$. The insets schematically illustrate the geometry and the isofrequency surfaces in wave-vector space. The polarization is evaluated at the discrete lattice sites $\boldsymbol{r}_{j}$ in the $x z$ plane. The other calculation parameters are the same as in Fig. 3.

approximation. Thus, the results of the two models, discrete and effective, may be compared only qualitatively.

The polarization (14) in Fig. 5 is evaluated at the discrete set of square lattice points $\boldsymbol{r}_{j}$ and the radiating dipole is located at the point $\boldsymbol{r}_{0}=0.5 a \hat{z}$; see the inset of Fig. 5(a). Figures 5(a) and 5(b) show the spatial distribution of the polarization Eq. (14) for the values of the effective dielectric constants of $\varepsilon_{z z}=2.5$ and $\varepsilon_{z z}=-1$, corresponding to Figs. 3 and 4 , respectively. In the case of the material with elliptic dispersion, the emission pattern is qualitatively the same as for the isotropic medium. The near field is concentrated at the emitter origin $\boldsymbol{r}=\boldsymbol{r}_{0}$, while the far field is emitted perpendicularly to the dipole axis. The pattern changes dramatically in the hyperbolic case [Fig. 5(b)]. The distribution has a distinct crosslike shape, typical for a hyperbolic medium. ${ }^{10,13}$ In the elliptic case, the only field singularity is that at the origin $R=\left|\boldsymbol{r}-\boldsymbol{r}_{0}\right|=0$. In the hyperbolic medium this singular point becomes a conical surface, where the field intensity is concentrated. Radiated waves are propagating within the cone $R^{2}>0$ and are evanescent outside this cone. The energy flow directions are normal to the isofrequency surfaces, so such a cone in $\boldsymbol{r}$ space is a direct counterpart of the hyperbolic dispersion curves in $\boldsymbol{k}$ space.

Comparing numerical and effective-medium results, Figs. 3, 4, and 5, we see that in the elliptic case the Green function is qualitatively the same as in the effective-medium approximation. Weak spatial modulation of the dipole moments $\left|p\left(\boldsymbol{r}_{j}\right)\right|$, seen in Fig. 3, is related to the deviations from the effective-medium theory Eq. (13), which, as mentioned above, is not completely valid for the point excitation. The distinct crosslike distribution of Fig. 4(a) is a fingerprint of the hyperbolic regime, similarly to the effective-medium approximation of Fig. 5(b). Comparing Figs. 5(b) and 4(a), we see that in the discrete case the singularity in the effectivemedium solution Eq. (13) at the conical surface $R=0$ is smeared out and even vanishes at large enough distances, where the effective-medium approximation also breaks down. This is qualitatively explained by the presence of the wavevector cutoff $\sim \pi / a$. The second striking difference between Fig. 4 and its effective-medium counterpart Fig. 5(b) is the strong spatial modulation of the distribution in the $y=0$ plane, manifested as vertical ripples. Such modulation is not explained by the effective-medium approximation of Fig. 5(b). The vertical ripples in Fig. 4(a) lying in the plane $y=0$ have period $t$ in the $x$ direction close to $2 a$. This allows us to interpret them as the interference pattern of the Bloch waves with wave vectors $k_{x}= \pm 2 \pi / t \equiv \pm \pi / a$, corresponding to the boundaries of the Brillouin zone. To check this hypothesis we have plotted in Fig. 6 the isofrequency curves in the $\Gamma-X$ and $\Gamma-M$ directions. Since $d k_{z} / d k_{x}=0$ at $k_{x}=\pi / a$ (the right panel of Fig. 6), there is a singularity in the density of waves, that propagate along the $x$ direction. This effect enhances the contribution of the wave vectors $k_{x}= \pm \pi / a$ and promotes the vertical ripples. Isofrequency curves calculated in the effective-medium approximation stay linear at the zone boundary (the thin lines in Fig. 6), so the singularity is absent and the ripples disappear. This has been additionally verified by substituting the effective-medium approximation [see Eq. (18) below] for the interaction constant in Eq. (4). Thus, the ripples can be thought of as the manifestation of the Van Hove band edge singularity ${ }^{54}$ in the Green function. ${ }^{55}$ For

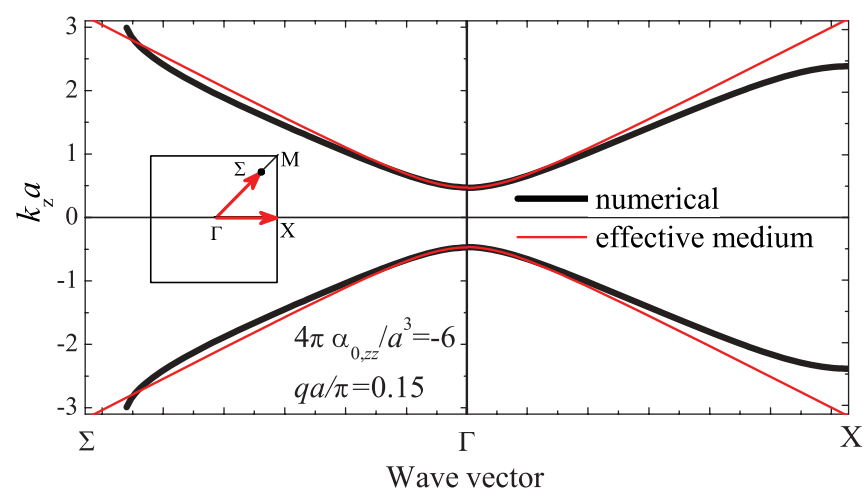

FIG. 6. (Color online) Isofrequency curves of the dipole lattice in the hyperbolic regime. Solid and thin lines correspond to the numerical calculation and the effective-medium approximation Eq. (11), respectively. The other parameters are the same as in Fig. 4. The inset schematically indicates the Brillouin zone of the square lattice; the point $\Sigma$ corresponds to $k_{x}=k_{y}=\pi /(\sqrt{2} a)$. 


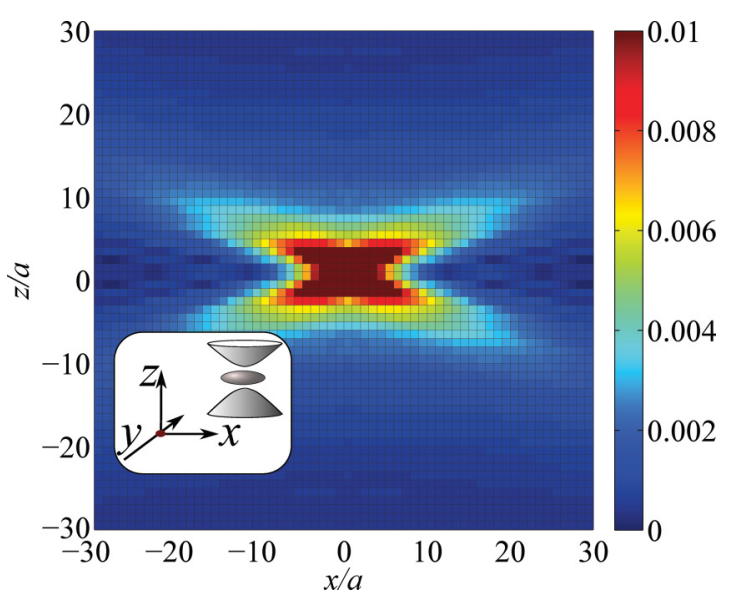

FIG. 7. (Color online) Spatial distribution of the dipole moments $\left|p_{z}(\boldsymbol{r})\right| / p_{0}$ in the mixed hyperbolic-elliptic regime with $\alpha_{0, z z}=$ $-1.3 a^{3} /(4 \pi)$. The insets schematically illustrate the geometry and the isofrequency surfaces in wave-vector space. The other calculation parameters are the same as in Fig. 5.

the $\Gamma-M$ direction the behavior of the isofrequency curves at the Brillouin zone edge is different [Fig. 6(b)] and the density of states singularity is absent, which explains the absence of ripples in the plane $y=x$ [Fig. 4(b)].

The discrete Green function calculated for the dipole polarizability $\alpha_{0, z z}=-1.3 a^{3} /(4 \pi)$, corresponding to the mixed elliptic-hyperbolic regime, is shown in Fig. 7. In this case the ripples are absent, because the isofrequency curves do not reach the Brillouin zone boundary $k_{x}= \pm \pi / a$; see the dashed curve in Fig. 2. Far-field emission along the $x$ direction is possible due to the modes with elliptic dispersion, providing a weak background to the field of the hyperbolic modes.

\section{PURCELL FACTOR}

Here we investigate the effect of the discreteness on the Purcell factor determining the characteristics of the spontaneous emission of the radiating dipole inside the material. The Purcell factor $f$ and the Lamb shift $l$ for the radiating dipole can be found from the Green function (8), evaluated at the dipole origin ${ }^{6,7,56}$ (see also Ref. 57):

$$
f+i l=1+\frac{3 i E_{z}\left(\boldsymbol{r}_{0}\right)}{2 q^{3} p_{0}} .
$$

Here, the dimensionless Lamb shift $l$ is in fact just a radiative correction to the resonance frequency of the radiating two-level system, normalized to its free-space decay rate. Equation (15) implies the weak-coupling regime, when the light-matter interaction is considered perturbatively. ${ }^{8}$ Gathering Eqs. (15), (8), and (4) together, we find the result in a compact form:

$$
f+i l=\frac{3 i}{2 q^{3}} \int_{(\mathrm{BZ})} \frac{V_{0} d^{3} k}{(2 \pi)^{3}} \frac{\left|G_{\boldsymbol{k}, z z}\left(\boldsymbol{r}_{0}\right)\right|^{2}}{1 / \alpha_{z z}-C(\boldsymbol{k})-i 0} .
$$

The frequency $\omega$, entering the wave vector $q$ in Eq. (16), is determined by the transition energy of the radiating dipole $\boldsymbol{d}_{0}$. It is clear from the structure of Eq. (16) that the Purcell factor is determined by the pole contribution, corresponding to emission of photons with the dispersion given by Eq. (9). We (a) Hyperbolic

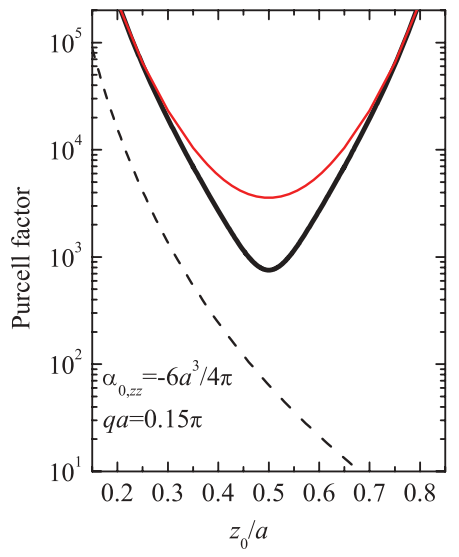

(b) Elliptic

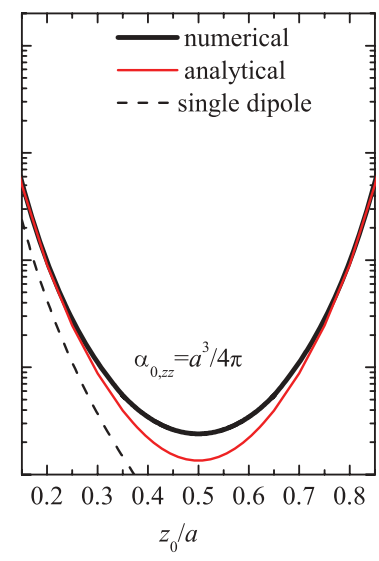

FIG. 8. (Color online) Purcell factor in the (a) hyperbolic and (b) elliptic regimes as a function of the source coordinate $z_{0}$ for $x_{0}=y_{0}=0$. The thick solid black, thin solid red, and dashed black curves correspond to numerical calculations, the analytical results of Eq. (20) (a) and Eq. (23) (b), and to a single dipole with corresponding polarizability [Eq. (25)], respectively. The other parameters as the same as in Fig. 4.

note that the first term on the right-hand side of Eq. (15) has canceled out in Eq. (16) with the pole contribution in the freespace Green function $G_{\boldsymbol{k}, z z}\left(\boldsymbol{r}_{0}\right)$ at $q=k$. We stress that, despite the classical formulation of the problem, the results for the emission rate and photon Green function may be equivalently obtained by a quantum-mechanical calculation, using either the Fermi golden rule ${ }^{7}$ or the local quantization framework. ${ }^{6}$

Numerical results for the dependence of the Purcell factor on the radiating dipole position within the unit cell of the structure are presented in Figs. 8 and 9. Figure 10 shows the frequency dependence of the Purcell factor. The figures demonstrate that the Purcell factor is much larger in the hyperbolic than in the elliptic regime. It is very sensitive to the dipole position and strongly increases when the dipole approaches the lattice nodes. Before discussing these results in more detail it is instructive to compared them with the analytical theory. (a) Infinite lattice

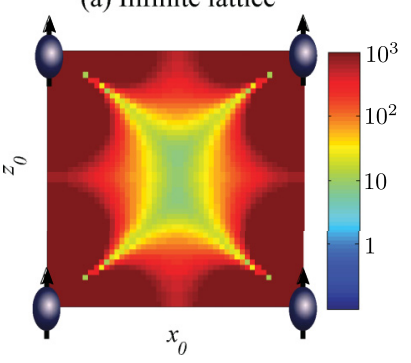

(b) Single dipole

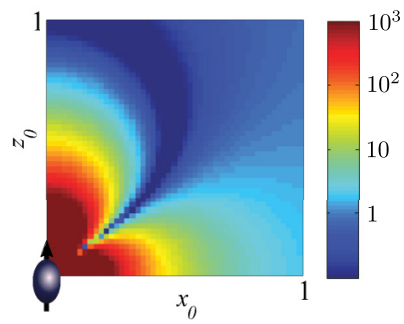

FIG. 9. (Color online) (a) Purcell factor in the hyperbolic medium as a function of the source position in the unit cell. (b) Calculation in the single-dipole approximation Eq. (25). The calculation was carried out at $y_{0}=0$ and the same other parameters as in Fig. 4. The radiating dipole coordinates change within the square $0 \leqslant x_{0} \leqslant 1,0 \leqslant z_{0} \leqslant 1$. The colors correspond to a logarithmic scale, identical for both panels. 

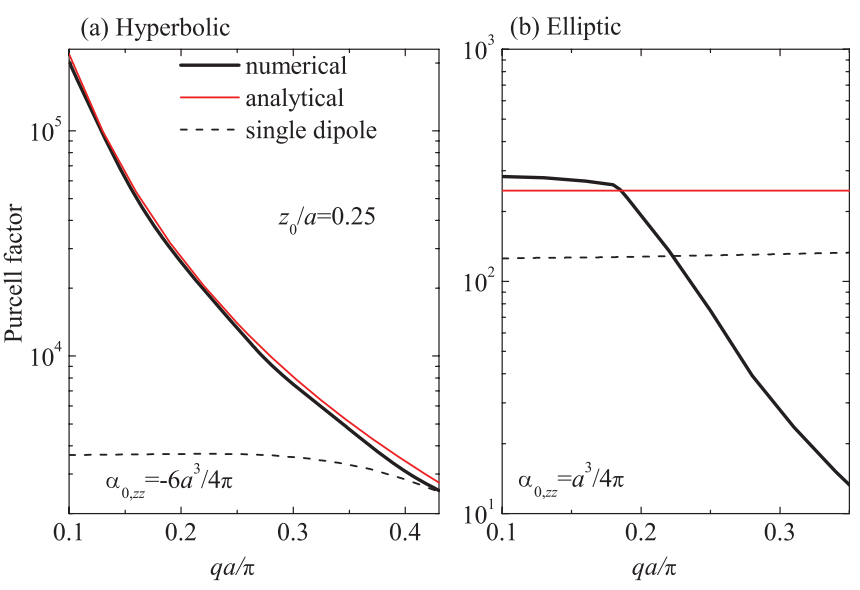

FIG. 10. (Color online) Purcell factor in the hyperbolic (a) and elliptic (b) regimes as a function of the frequency qa for $\boldsymbol{r}_{0}=0.25 a \hat{z}$. The notation and other parameters are the same as in Fig. 8.

\section{A. Analytical results}

Here we focus on the Purcell factor in the quasistatic limit $q \ll \pi / a$. Equation (16) can be then reduced to

$$
f=\frac{3}{2 q^{3}} \frac{V_{0}\left|G_{z z, \text { stat }}\left(\boldsymbol{r}_{0}\right)\right|^{2}}{(2 \pi)^{2}} \int d k_{x} d k_{y}\left|\frac{d C(\boldsymbol{k})}{d k_{z}}\right|_{k_{z}\left(k_{x}, k_{y}\right)}^{-1},
$$

where the interaction constant in the effective-medium approximation reads 45

$$
C(\boldsymbol{k})=\frac{4 \pi}{V_{0}} \frac{q^{2}-k_{z}^{2}}{k^{2}-q^{2}}+\frac{4 \pi}{3 V_{0}}+\frac{2 i q^{3}}{3} .
$$

The integral over $k_{z}$ in Eq. (16) is determined by the residues of the waves with $\pm k_{z}\left(k_{x}, k_{y}\right)$, which are the solutions of Eq. (9) at a given frequency. The integration over $k_{x}$ and $k_{y}$ in Eq. (17) is restricted to those vectors within the two-dimensional Brillouin zone for which such a solution exists. The quantity $G_{z z \text {,stat }}\left(\boldsymbol{r}_{0}\right)$ in Eq. (17) is the quasistatic approximation of the Green function Eq. (6): $\left.G_{z z \text {,stat }}\left(\boldsymbol{r}_{0}\right) \equiv G_{\boldsymbol{k}, z z}\left(\boldsymbol{r}_{0}\right)\right|_{\boldsymbol{k}=\mathbf{0}, q=0}$. The value of $G_{z z \text {,stat }}$ is determined by the near field of the lattice dipoles, closest to the radiating one. The maximum Purcell factor can then be expected when the source is located at the vertical edge of the elementary cell, i.e., $x_{0}=y_{0}=0, z_{0} \neq 0$. In this case $G_{z z \text {,stat }}\left(z_{0}\right)$ can be reduced to

$$
G_{z z, \text { stat }}\left(z_{0}\right) \approx \frac{2}{z_{0}^{3}}+\frac{2}{\left(a-z_{0}\right)^{3}}
$$

and grows dramatically when the emitter approaches the lattice node. Evaluating the derivative in Eq. (17) by means of Eq. (18) and performing the integration, we obtain the analytical result for the Purcell factor

$$
f_{\text {hyp }}=\frac{(\varepsilon-1)^{2}}{32 \pi^{2}}\left(\frac{k_{z, \text { max }}}{q}\right)^{3}\left|V_{0} G_{z z \text {,stat }}(z)\right|^{2}
$$

in the hyperbolic medium. Here, $k_{z, \max } \gg q$ is the cutoff for the wave vector $k_{z}$ due to the finite extent of the Brillouin zone. Its value depends on the effective dielectric constant,

$$
k_{z, \max } \approx \begin{cases}\frac{\pi}{a}, & -1 \leqslant \varepsilon \leqslant 0, \\ \frac{\pi}{a \sqrt{\left|\varepsilon_{z z}\right|},} & \varepsilon_{z z} \leqslant-1 .\end{cases}
$$

Thus, Eq. (20) provides a compact analytical result for the Purcell factor in the lossless hyperbolic medium. Its general structure can be understood as follows: the factor $\left(k_{z, \max } / q\right)^{3} \sim$ $1 /(q a)^{3}$ describes the enhancement of the photonic density of states as compared to the vacuum. The second factor $\left|V_{0} G_{z z \text {,stat }}(z)\right|^{2}$ reflects the coordinate dependence of the Purcell factor, governed by the near field of the neighboring dipoles. ${ }^{58}$ Near the lattice nodes Eq. (20) can be simplified to

$$
f_{\text {hyp }}(q, z \rightarrow 0) \approx \frac{\pi(\varepsilon-1)^{2} a^{3}}{8 q^{3}|z|^{6}},
$$

where we assumed that $|\varepsilon| \leqslant 1$.

A similar calculation can also be performed in the elliptic case when $\varepsilon_{z z}>0$. It should be noted that in the effectivemedium approximation, the Purcell factor for the axial dipole in the elliptic medium is unity, independently of the value of $\varepsilon_{z z} \cdot{ }^{32}$ Local field corrections can still promote a high decay rate. The answer reads

$$
f_{\text {ell }}=\left|G_{\text {stat }, z z}\right|^{2}\left|\frac{V_{0}(\varepsilon-1)}{4 \pi}\right|^{2} .
$$

This expression depends on the local field intensity, similarly to Eq. (20), but is smaller by the factor

$$
\frac{f_{\text {hyp }}}{f_{\text {ell }}}=\frac{k_{z, \max }^{3}}{2 q^{3}},
$$

since the density of states in the elliptic medium is smaller. In order to distinguish between the local field effects and the collective effects due to density of states enhancement in the medium it is instructive to analyze also the Purcell factor for a source located in vacuum near a single dipole at the point $\boldsymbol{r}=\mathbf{0}$. The result reads ${ }^{2,48}$

$$
f_{1}=1+\frac{3}{2 q^{3}} \operatorname{Im}\left[\alpha_{z z} G_{0, z z}^{2}\left(\boldsymbol{r}_{0}\right)\right]
$$

here, the second term is the field of the emitter reflected from the dipole. In the quasistatic limit $q \rightarrow 0$, Eq. (25) reduces to

$$
f_{1}=\left(1+\frac{\alpha_{0, z z}}{\left|z^{3}\right|}\right)^{2} .
$$

Both Eqs. (22) and (26) demonstrate divergency when $z$ tends to zero. However, their dependence on the wave vector $q$ is quite different: Eq. (22) diverges as $1 / q^{3}$ at small $q$, while Eq. (26) does not depend on $q$ at all. This divergency is a characteristic effect increasing photonic density of states in a hyperbolic medium. ${ }^{20,26,32}$

\section{B. Numerical results}

Now we discuss the calculated dependence of the Purcell factor on the source position and on the transition frequency $\omega=c q$, shown in Figs. 8, 9, and 10. The calculations confirm the singular behavior of the Purcell factor in the hyperbolic case when the source approaches the lattice nodes [the solid curve in Fig. 8(a)]. The singularity is excellently described by the analytical Eq. (20) (the thin red curve). The interaction of the emitter with a single dipole [Eq. (25)] provides substantially smaller enhancements (the dashed black curve), although 


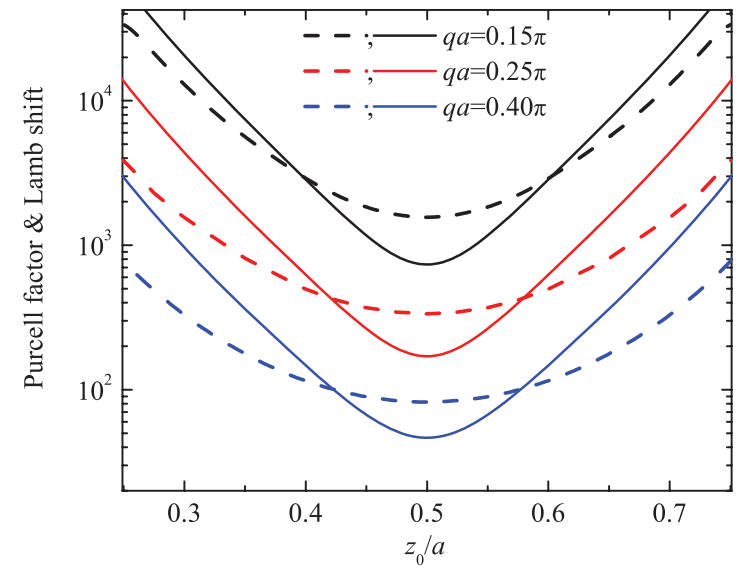

FIG. 11. (Color online) Purcell factor (solid lines) and Lamb shift (dashed lines) dependence on the coordinate $z_{0}$ of the source in the unit cell for different values of $q a$. The calculation parameters are the same as in Fig. 4.

it also diverges at $z=0$ and $z=a$. Additional comparison of the exact calculation in the medium with the result for a single dipole is presented by the Purcell factor dependence on the two coordinates $x$ and $z$ in the unit cell, shown in Fig. 9. Equation (25), taking into account only a single lattice dipole at the point $\boldsymbol{r}=\mathbf{0}$, satisfactorily reproduces the Purcell factor pattern near this point. The corresponding two-dimensional plot of the Purcell factor in the quadrant $0 \leqslant x, y \leqslant 1$ is shown on Fig. 9(b). Comparing the two panels of Fig. 9 we see that near the corner $\boldsymbol{r}=\mathbf{0}$ the angular dependence is approximately given by $\left(3 \cos ^{2} \theta-1\right)^{2}$, where $\theta$ is the polar angle. Still, the single-dipole model with corresponding polarizability $\alpha_{0, z z}=-6 a^{3} /(4 \pi)$ considerably underestimates the absolute values of the Purcell factor. The situation is different in the elliptic case, where all three approaches, namely, numerical calculation according to Eq. (16), the single-dipole model (25) with $\alpha_{0, z z}=a^{3} /(4 \pi)$, and the analytical model (23) provide similar results [Fig. 8(b)].

The failure of the single-dipole model in the hyperbolic medium is also revealed in the frequency dependence of the Purcell factor [Fig. 10(a)]. The dashed curve, calculated for a single dipole, tends to the limit Eq. (26), which is frequency independent. However, the Purcell factor in the hyperbolic medium diverges at low frequencies as $1 / q^{3}$, according to Eq. (22). The Lamb shift $l$, calculated in the hyperbolic medium for different values of $q a$ is presented in Fig. 11 by the dashed curves. The Lamb shift is of the same order as the Purcell factor (dashed curve) and has similar near-field singularities at the node sites.
To summarize, Figs. 8-11 underline the importance of the local field effects in the hyperbolic medium and confirm the collective origin of the spontaneous emission enhancement.

\section{CONCLUSIONS}

We have developed the analytical theory of light-matter coupling in discrete hyperbolic metamaterials in the framework of the discrete model of a cubic lattice of uniaxial resonant dipoles. We have calculated the Purcell factor, Lamb shift, and Green function for such a discrete model, and demonstrated that the optimal emitter position is in the local field maxima, close to the lattice nodes. We have demonstrated that the density of states is drastically enhanced in the hyperbolic regime as compared to other cases including vacuum, the elliptic regime, or the single-resonant-dipole case. As a result, a huge number of lattice dipoles are efficiently excited by the emitter, which has been visualized by calculating the Green function of the lattice. The Green function has the shape of a cone: the field propagates along the directions close to the symmetry axis $z$ and decays in the $x y$ plane. The discrete character of the problem results in strong spatial modulation of the Green function.

Experimental state-of-the art metamaterials are characterized by substantially lower absolute values of the Purcell factor than those calculated here. This is mainly due to the point-dipole approximation we have utilized: as the distance to the scatterers becomes comparable to their sizes, higher-order multipoles must be accounted for. This will inevitably reduce the local field and suppress the Purcell factor. Finally, losses are always present and can significantly influence the discussed numerical answers. All such effects will be of high importance in the optical frequency range. Indeed, e.g., for dielectric quantum microcavities the value of 10 for the Purcell factor is already considered as large.$^{59}$ For lower frequencies one can expect a much stronger effect: a huge enhancement factor $>10^{18}$ was claimed already in the original Purcell paper. ${ }^{1}$ Thus, we believe that our results will remain qualitatively correct for more complex settings, and they provide an important insight into the rapidly developing physics of hyperbolic metamaterials.

\section{ACKNOWLEDGMENTS}

This work has been supported by the Ministry of Education and Science of the Russian Federation, the Dynasty Foundation, the Russian Foundation for Basic Research, the European project POLAPHEN, EPSRC (UK), and the Australian Research Council. The authors acknowledge useful discussions with C. R. Simovski.
${ }^{1}$ E. M. Purcell, Phys. Rev. 69, 681 (1946).

${ }^{2}$ L. Novotny and B. Hecht, Principles of Nano-Optics (Cambridge University Press, New York, 2006).

${ }^{3}$ Z. Jacob and V. M. Shalaev, Science 334, 463 (2011).

${ }^{4}$ H. N. S. Krishnamoorthy, Z. Jacob, E. Narimanov, I. Kretzschmar, and V. M. Menon, Science 336, 205 (2012).
${ }^{5}$ H. Gibbs, G. Khitrova, and S. Koch, Nat. Photonics 5, 273 (2011).

${ }^{6}$ W. Vogel and D.-G. Welsch, Quantum Optics (Wiley, Weinheim, 2006).

${ }^{7}$ E. L. Ivchenko, Optical Spectroscopy of Semiconductor Nanostructures (Alpha Science International, Harrow, UK, 2005). 
${ }^{8}$ A. Kavokin, J. Baumberg, G. Malpuech, and F. Laussy, Microcavities (Clarendon Press, Oxford, 2006).

${ }^{9}$ M. G. Silveirinha and S. I. Maslovski, Phys. Rev. B 85, 155125 (2012).

${ }^{10}$ L. Felsen and N. Marcuvitz, Radiation and Scattering of Waves (Wiley Interscience, New York, 2003).

${ }^{11}$ I. V. Lindell, S. A. Tretyakov, K. I. Nikoskinen, and S. Ilvonen, Microwave Opt. Technol. Lett. 31, 129 (2001).

${ }^{12}$ D. R. Smith and D. Schurig, Phys. Rev. Lett. 90, 077405 (2003).

${ }^{13}$ R. K. Fisher and R. W. Gould, Phys. Rev. Lett. 22, 1093 (1969).

${ }^{14}$ J. Sun, J. Zhou, B. Li, and F. Kang, Appl. Phys. Lett. 98, 101901 (2011).

${ }^{15}$ G. A. Wurtz, W. Dickson, D. O'Connor, R. Atkinson, W. Hendren, P. Evans, R. Pollard, and A. V. Zayats, Opt. Express 16, 7460 (2008).

${ }^{16}$ M. A. Noginov, Y. A. Barnakov, G. Zhu, T. Tumkur, H. Li, and E. E. Narimanov, Appl. Phys. Lett. 94, 151105 (2009).

${ }^{17}$ C. R. Simovski, P. A. Belov, A. V. Atrashchenko, and Y. S. Kivshar, Adv. Mater. (2012), doi: 10.1002/adma.201200931.

${ }^{18}$ J. Elser, V. A. Podolskiy, I. Salakhutdinov, and I. Avrutsky, Appl. Phys. Lett. 90, 191109 (2007).

${ }^{19}$ A. A. Orlov, P. M. Voroshilov, P. A. Belov, and Y. S. Kivshar, Phys. Rev. B 84, 045424 (2011).

${ }^{20}$ Z. Jacob, J. Kim, G. V. Naik, A. Boltasseva, E. E. Narimanov, and V. M. Shalaev, Appl. Phys. B 100, 215 (2010).

${ }^{21}$ M. A. Noginov, H. Li, Y. A. Barnakov, D. Dryden, G. Nataraj, G. Zhu, C. E. Bonner, M. Mayy, Z. Jacob, and E. E. Narimanov, Opt. Lett. 35, 1863 (2010).

${ }^{22}$ X. Ni, S. Ishii, M. D. Thoreson, V. M. Shalaev, S. Han, S. Lee, and A. V. Kildishev, Opt. Express 19, 25242 (2011).

${ }^{23}$ J. Kim, V. P. Drachev, Z. Jacob, G. V. Naik, A. Boltasseva, E. E. Narimanov, and V. M. Shalaev, Opt. Express 20, 8100 (2012).

${ }^{24}$ C. L. Cortes, W. Newman, S. Molesky, and Z. Jacob, J. Opt. 14, 063001 (2012).

${ }^{25}$ Z. Jacob, I. Smolyaninov, and E. Narimanov, arXiv:0910.3981.

${ }^{26}$ S. I. Maslovski and M. G. Silveirinha, Phys. Rev. A 83, 022508 (2011).

${ }^{27}$ I. Iorsh, A. Poddubny, A. Orlov, P. Belov, and Y. S. Kivshar, Phys. Lett. A 376, 185 (2012).

${ }^{28}$ H. Xie, P. Leung, and D. Tsai, Solid State Commun. 149, 625 (2009).

${ }^{29}$ O. Kidwai, S. V. Zhukovsky, and J. E. Sipe, Opt. Lett. 36, 2530 (2011).

${ }^{30}$ O. Kidwai, S. V. Zhukovsky, and J. E. Sipe, Phys. Rev. A 85, 053842 (2012).

${ }^{31}$ W. Yan, M. Wubs, and N. Asger Mortensen, arXiv:1204.5413.

${ }^{32}$ A. N. Poddubny, P. A. Belov, and Y. S. Kivshar, Phys. Rev. A 84, 023807 (2011).
${ }^{33}$ E. L. Ivchenko, Y. Fu, and M. Willander, Phys. Solid State 42, 1756 (2000).

${ }^{34}$ Y. Zeng, Y. Fu, X. Chen, W. Lu, and H. Ågren, Phys. Rev. B 74, 115325 (2006)

${ }^{35}$ I. H. Deutsch, R. J. C. Spreeuw, S. L. Rolston, and W. D. Phillips, Phys. Rev. A 52, 1394 (1995).

${ }^{36}$ D. V. van Coevorden, R. Sprik, A. Tip, and A. Lagendijk, Phys. Rev. Lett. 77, 2412 (1996).

${ }^{37}$ J. A. Klugkist, M. Mostovoy, and J. Knoester, Phys. Rev. Lett. 96, 163903 (2006).

${ }^{38}$ H. Miyake, G. A. Siviloglou, G. Puentes, D. E. Pritchard, W. Ketterle, and D. M. Weld, Phys. Rev. Lett. 107, 175302 (2011).

${ }^{39}$ Y. Kagan, Hyperfine Interact. 123, 83 (1999).

${ }^{40}$ E. M. Purcell and C. R. Pennypacker, Astrophys. J. 186, 705 (1973).

${ }^{41}$ A. Rahmani, P. C. Chaumet, and G. W. Bryant, Opt. Express 18, 8499 (2010).

${ }^{42}$ D. R. Smith, W. J. Padilla, D. C. Vier, S. C. Nemat-Nasser, and S. Schultz, Phys. Rev. Lett. 84, 4184 (2000).

${ }^{43}$ P. A. Belov and C. R. Simovski, Phys. Rev. E 72, 026615 (2005).

${ }^{44}$ I. V. Shadrivov, A. A. Zharov, N. A. Zharova, and Y. S. Kivshar, Photonics Nanostruct. Fundam. Applic. 4, 69 (2006).

${ }^{45}$ M. G. Silveirinha and P. A. Belov, Phys. Rev. B 77, 233104 (2008).

${ }^{46}$ I. Sersic, M. A. van de Haar, F. B. Arango, and A. F. Koenderink, Phys. Rev. Lett. 108, 223903 (2012).

${ }^{47}$ B. Sauviac, C. R. Simovsky, and S. A. Tretyakov, Electromagnetics 24, 317 (2004).

${ }^{48}$ P. de Vries, D. V. van Coevorden, and A. Lagendijk, Rev. Mod. Phys. 70, 447 (1998).

${ }^{49}$ J. Korringa, Physica 13, 392 (1947).

${ }^{50}$ F. W. de Wette and G. E. Schacher, Phys. Rev. 137, A78 (1965).

${ }^{51}$ L. Landau and E. Lifshitz, Electrodynamics of Continuous Media (Pergamon, New York, 1974).

${ }^{52}$ R. J. Pollard, A. Murphy, W. R. Hendren, P. R. Evans, R. Atkinson, G. A. Wurtz, A. V. Zayats, and V. A. Podolskiy, Phys. Rev. Lett. 102, 127405 (2009).

${ }^{53}$ A. Savchenko and O. Savchenko, Tech. Phys. 50, 1366 (2005).

${ }^{54}$ C. Kittel, Introduction to Solid State Physics (Wiley, New York, 1996).

${ }^{55}$ R. H. Swendsen and H. Callen, Phys. Rev. B 6, 2860 (1972).

${ }^{56}$ M. Glazov, E. Ivchenko, A. Poddubny, and G. Khitrova, Phys. Solid State 53, 1753 (2011).

${ }^{57}$ X.-H. Wang, Y. S. Kivshar, and B.-Y. Gu, Phys. Rev. Lett. 93, 073901 (2004).

${ }^{58}$ A. Rahmani, P. C. Chaumet, and G. W. Bryant, Opt. Lett. 27, 430 (2002).

${ }^{59}$ S. Reitzenstein and A. Forchel, J. Phys. D 43, 033001 (2010). 